\title{
Sum rules and physical bounds in electromagnetic theory
}

\author{
Mats Gustafsson ${ }^{\# 1}$, Daniel Sjöberg ${ }^{\# 2}$, Anders Bernland ${ }^{\# 3}$, Gerhard Kristensson ${ }^{* 4}$, Christian Sohl \#5 \\ \# Department of Electrical and Information Technology, Lund University \\ Box 118, SE-221 00 Lund, Sweden \\ ${ }^{1}$ mats.gustafssondeit.lth.se \\ ${ }^{2}$ daniel.sjoberg@eit.lth.se \\ 3 anders.bernlandeeit.lth.se \\ ${ }^{4}$ gerhard.kristenssondeit.lth.se \\ ${ }^{5}$ christian.sohldeit.lth.se
}

\begin{abstract}
Sum rules are useful in many branches of physics and engineering as they relate all spectrum parameter values with their asymptotic expansions. Properties of the dynamic response can hence be inferred by the, in many cases much simpler, static response. This has e.g., been used for lossless matching networks, radar absorbers, extinction cross section, partial realized gain of antennas, high-impedance surfaces, transmission cross section, transmission coefficients, and temporal dispersion of metamaterials. Here, several sum rules and their associated physical bounds are reviewed and it is shown that integral identities for Herglotz functions offer a unified approach in deriving them.
\end{abstract}

\section{INTRODUCTION}

Sum rules are e.g., useful in deriving physical bounds as they relate dynamic properties of a parameter with static and high-frequency values. These type of identities and physical bounds are of great interest in many areas of electromagnetic theory. They provide physical insight of the relation between design parameters. They are also useful in optimization of $e . g$., antennas, metamaterials, and radar absorbers as they provide upper bounds on the design. In this paper, sum rules for various scattering, antenna, and material problems are reviewed. It is shown that integral identities for Herglotz functions offer a unified approach for them.

\section{HERGLOTZ FUNCTIONS AND INTEGRAL IDENTITIES}

The sum rules presented here are based on integral identities for Herglotz functions [1,2]. Herglotz functions (also known as Nevanlinna or Pick functions and closely related to positive real functions [3]), $h(z)$, are analytic and $\operatorname{Im}\{h(z)\} \geq 0$ for $\operatorname{Im} z>0$. They are often found in linear, passive, and causal systems [2,3]. The identities

$$
\frac{2}{\pi} \int_{0}^{\infty} \frac{\operatorname{Im} h(x)}{x^{2 p}} \mathrm{~d} x=a_{2 p-1}-b_{2 p-1}
$$

for $p=1-M, 2-M, \ldots, N$ are valid for all symmetric Herglotz functions $h(z)=-h^{*}\left(-z^{*}\right)$ having the asymptotic expansions

$$
h(z)=\sum_{n=-1}^{2 N-1} a_{n} z^{n}+\mathrm{o}\left(z^{2 N-1}\right) \quad \text { as } z \hat{\rightarrow} 0
$$

and

$$
h(z)=\sum_{m=1-2 M}^{1} b_{m} z^{m}+\mathrm{o}\left(z^{1-2 M}\right) \text { as } z \hat{\rightarrow} \infty,
$$

where $\hat{\rightarrow}$ means limits in some sector $0<\alpha<\arg z<\pi-\alpha$, see [2] for a proof and details of the integral in (1).

The identities (1) are instrumental for sum rules in electromagnetics [4-8], antennas [9,10], optics [11], circuit theory [12], and many other branches of physics [13]. Here, several sum rules and their associated physical bounds are reviewed. They are used to illustrate the unified approach that follow from the identities (1).

\section{SCATTERING BY FINITE OBJECTS}

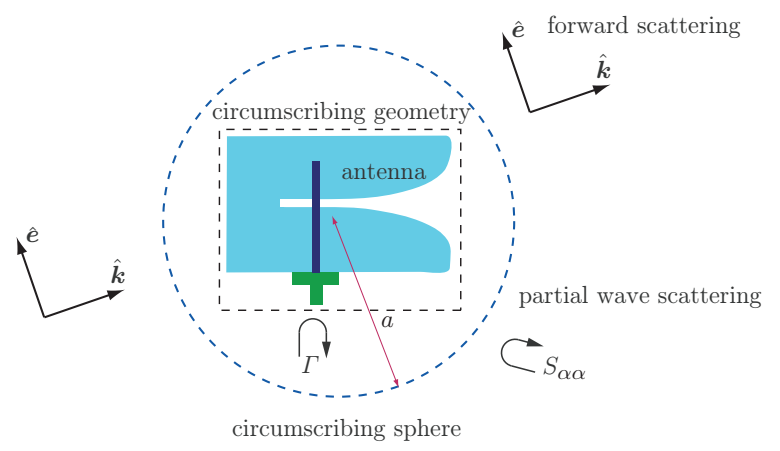

Fig. 1. Illustration of forward scattering of a plane wave in the direction $\hat{\boldsymbol{k}}$ and linear polarization $\hat{\boldsymbol{e}}$, partial wave (mode) scattering, and transmission line scattering for finite objects and antennas.

There are several scattering setups for finite scatterers and antennas, e.g., forward scattering, partial wave (mode) scattering, transmission line scattering, see Fig. 1.

\section{A. Extinction cross section}

Forward scattering and the associated sum rule for the extinction cross section are analyzed in [5] and we refer to this paper for details. Causality is ensured in the forward scattering case, i.e., a plane wave impinging on the object and the scattered co-polarized component is determined in the 
forward direction. This forward scattered field defines a causal function if the wavefront velocity is lower in the object than in the surrounding medium. This is obviously the case for object in free space as the wave front velocity cannot exceed the speed of light. It is however in general not true in acoustic scattering [13]. The derivation in [5] is based on the optical theorem that defines a Herglotz function, $h_{\text {ext }}(k)$, such that $\operatorname{Im} h_{\text {ext }}(k)=\sigma_{\text {ext }}(k)$, where $\sigma_{\text {ext }}$ denotes the extinction cross section and $k$ is the wavenumber.

The Herglotz function $h_{\text {ext }}(k)$ has the low-frequency expansion

$$
h_{\mathrm{ext}}(k)=k\left(\hat{\boldsymbol{e}} \cdot \gamma_{\mathrm{e}} \cdot \hat{\boldsymbol{e}}+(\hat{\boldsymbol{k}} \times \hat{\boldsymbol{e}}) \cdot \gamma_{\mathrm{m}} \cdot(\hat{\boldsymbol{k}} \times \hat{\boldsymbol{e}})\right)+\mathrm{o}(k)
$$

as $k \rightarrow 0$, where $\gamma_{\mathrm{e}}$ and $\gamma_{\mathrm{m}}$ denote the electric and magnetic polarizability dyadics, respectively. The low-frequency expansion (4) shows that $N=1$ in (2), implying that there is a $p=1$ identity in (1), i.e.,

$$
\frac{2}{\pi} \int_{0}^{\infty} \frac{\sigma_{\text {ext }}(k)}{k^{2}} \mathrm{~d} k=\hat{\boldsymbol{e}} \cdot \gamma_{\mathrm{e}} \cdot \hat{\boldsymbol{e}}+(\hat{\boldsymbol{k}} \times \hat{\boldsymbol{e}}) \cdot \gamma_{\mathrm{m}} \cdot(\hat{\boldsymbol{k}} \times \hat{\boldsymbol{e}}) .
$$

This identity was first derived for spheroidal dielectrics [14] and generalized to arbitrary objects in $[5,15]$. Note that the $b_{1} \geq 0$ coefficient in (3) vanishes in forward scattering [5, 15].

The polarizability dyadics in the right-hand side of (5) are determined from the solution of the Laplace equation. There are also several geometries with closed form expressions, e.g., a dielectric sphere with static relative permittivity $\epsilon_{\mathrm{S}}$ and radius $a$ has

$$
\gamma_{\mathrm{e}}=4 \pi a^{3} \frac{\epsilon_{\mathrm{s}}-1}{\epsilon_{\mathrm{s}}+2} \mathbf{I} \leq \gamma_{\infty}=4 \pi a^{3} \mathbf{I} \quad \text { and } \gamma_{\mathrm{m}}=\mathbf{0}
$$

where $\gamma_{\infty}$ is the high-contrast polarizability.

As an example consider spherical scatterers composed by either aluminum $(\mathrm{Al})$, silver $(\mathrm{Ag})$, gold $(\mathrm{Au})$, or copper $(\mathrm{Cu})$, using the permittivity models in [16]. The extinction cross sections for spheres with radius $a=0.1 \mu \mathrm{m}$ are depicted in Fig. 2 as functions of the wavelength $\lambda=2 \pi / k$. It is observed that $\sigma_{\text {ext }}$ is large compared to the cross section area $\pi a^{2}$ at some resonance wavelengths and that $\sigma_{\text {ext }}$ is small for $\lambda>$ $1 \mu \mathrm{m}$. The spheres are metallic and have the polarizabilities $\gamma_{\mathrm{e}}=\gamma_{\infty}=4 \pi a^{3} \mathbf{I}$ and $\gamma_{\mathrm{m}}=\mathbf{0}$. Note that the identity (5) gives the bound

$$
\frac{\left(\lambda_{2}-\lambda_{1}\right)}{\pi^{2}} \min _{\lambda \in\left[\lambda_{1}, \lambda_{2}\right]} \sigma_{\text {ext }}(\lambda) \leq 4 \pi a^{3}
$$

and with $\lambda_{1}=0$ it simplifies to the bound

$$
\lambda_{2} \min _{\lambda<\lambda_{2}} \frac{\sigma_{\text {ext }}(\lambda)}{\pi a^{2}} \leq 4 \pi^{2} a
$$

that e.g., shows that $\min _{\lambda<\lambda_{2}} \frac{\sigma_{\text {ext }}(\lambda)}{\pi a^{2}} \leq 4$ for $\lambda_{2}=1 \mu \mathrm{m}$ and $a=0.1 \mu \mathrm{m}$.

\section{Planar SURfaCES}

Planar periodic structures are important in electromagnetic applications such as absorbers, frequency selective surfaces, and high impedance surfaces. The electromagnetic properties

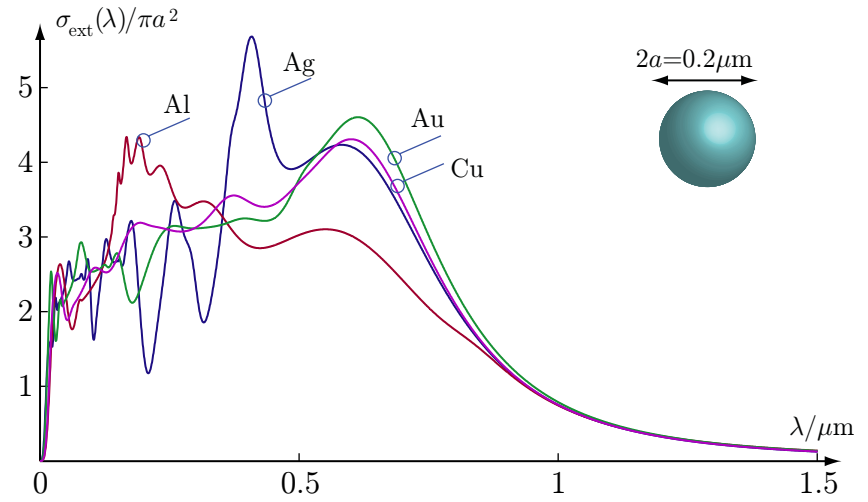

Fig. 2. Extinction cross sections for aluminum (Al), silver (Ag), gold $(\mathrm{Au})$, and copper $(\mathrm{Cu})$ spheres with radius $a=0.1 \mu \mathrm{m}$ as function of the wavelength $\lambda=2 \pi / k$.

are often represented by the reflection and transmission coefficients. For simplicity, the cases with normal incident plane waves are considered here.

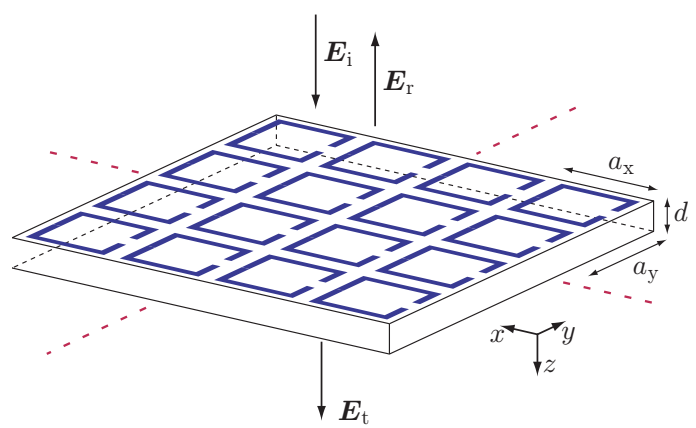

Fig. 3. Scattering by a planar periodic structure with reflected co-polarized field $\boldsymbol{E}_{\mathrm{r}}=\Gamma \boldsymbol{E}_{\mathrm{i}}$ and transmitted co-polarized field $\boldsymbol{E}_{\mathrm{t}}=T \boldsymbol{E}_{\mathrm{i}}$.

\section{A. Transmission blockages}

Consider a dielectric structure with a periodic pattern of metallic inclusions such as the split ring resonators in Fig. 3. This is a low-pass frequency selective surface, i.e., low frequency waves do not interact with the structure. This case is analyzed in [7], and we refer to this paper for details. It is first observed that the transmitted field cannot precede the incident field. This implies that the transmission coefficient is analytic in the upper complex half plane. The transmission coefficient is also bounded by unity for passive structures. A conformal mapping of the unit circle to the upper complex half plane finally defines a Herglotz function.

The logarithm $-\ln |T|$ is often considered as the amplitude of the transmission coefficient is important. Here, it is first necessary to remove the zeros $k_{n}$ of $T$ with $\operatorname{Im} k_{n}>0$, see [2, $7,12]$ for details. This defines the Herglotz function

$$
h(k)=-\mathrm{i} \ln \left(T(k) \prod_{n=1}^{N} \frac{k_{n}^{*}-k}{k_{n}-k}\right),
$$

where an asterisk denotes the complex conjugate, see [2] for the case with $N=\infty$. The low-frequency expansion of $T$ is 
$T \sim 1+\mathrm{i} k \hat{\boldsymbol{e}} \cdot \gamma_{\mathrm{e}} \cdot \hat{\boldsymbol{e}} /(2 A)$, where $A$ denotes the area of the unit cell, see $[7,17]$, that gives

$$
-\mathrm{i} \ln T(k) \sim k \frac{\hat{\boldsymbol{e}} \cdot \gamma_{\mathrm{e}} \cdot \hat{\boldsymbol{e}}}{2 A}
$$

and the low-frequency expansion of $h$ as

$$
h(k)=k \frac{\hat{\boldsymbol{e}} \cdot \gamma_{\mathrm{e}} \cdot \hat{\boldsymbol{e}}}{2 A}+k \sum_{n=1}^{N} \frac{1}{k_{n}}+\mathrm{o}(k) \quad \text { as } k \hat{\rightarrow} 0 .
$$

It is noted that (1) offer a $p=1$ sum rule, i.e.,

$$
\frac{2}{\pi} \int_{0}^{\infty} \frac{1}{k^{2}} \ln \frac{1}{|T(k)|} \mathrm{d} k \leq \frac{\hat{\boldsymbol{e}} \cdot \gamma_{\mathrm{e}} \cdot \hat{\boldsymbol{e}}}{2 A},
$$

where $\operatorname{Im} k_{n}>0$ is used to rewrite the identity (1) into an inequality.

\section{B. Absorbers}

Absorbers are often supported by a perfectly conducting ground plane. The performance of absorbers is quantified by the magnitude of the reflection coefficient. For simplicity, the case with a layer with a homogeneous permeability and a periodic permittivity is considered. This gives the lowfrequency expansion $[4,17]$

$$
\Gamma(k)=1+\mathrm{i} 2 \mu_{\mathrm{s}} k d+\mathrm{o}(k),
$$

where $d$ is the thickness of the layer and $\mu_{\mathrm{s}}$ is the static relative permeability of the layer. Remove the zeros of $\Gamma(k)$ as in (9) to define the Herglotz function

$$
h(k)=-\mathrm{i} \ln \left(\Gamma(k) \prod_{n=1}^{N} \frac{k_{n}^{*}-k}{k_{n}-k}\right)
$$

that has the low-frequency expansion

$$
h(k)=2 \mu_{\mathrm{s}} k d+\mathrm{o}(k) \quad \text { as } k \hat{\rightarrow} 0 .
$$

The identities (1) show that there is a $p=1$ sum rule, i.e.,

$$
\frac{2}{\pi} \int_{0}^{\infty} \frac{1}{k^{2}} \ln \frac{1}{|\Gamma(k)|} \mathrm{d} k \leq 2 \mu_{\mathrm{s}} d .
$$

This identity was derived by Rozanov [4] and is used to determine physical bounds on radar absorbers.

\section{High impedance surfaces}

High-impedance surfaces are also based on periodic structures above a perfectly conducting ground plane. The structures have ideally $\Gamma \approx 1$, i.e., the reflection does not change the phase of the wave. Use the impedance, $Z$, of the surface to characterize the surface. The reflection coefficient of a surface is $\Gamma=\left(Z-\eta_{0}\right) /\left(Z+\eta_{0}\right)$, where $\eta_{0}$ denotes the free space impedance. The low-frequency expansion of $\Gamma$ is identical to the absorber case (13) and gives the corresponding expansion of the impedance

$$
Z(k)=\eta_{0} \frac{1-\Gamma}{1+\Gamma} \sim-\mathrm{i} k d \mu_{\mathrm{s}} \eta_{0} \quad \text { as } k \hat{\rightarrow} 0 .
$$

It is possible to derive several identities for the impedance. However, to derive a sum rule that is particularly suitable for this case it is convenient to introduce the Herglotz function [8]

$$
h_{\Delta}(z)=\frac{1}{\pi} \int_{-\Delta}^{\Delta} \frac{1}{\xi-z} \mathrm{~d} \xi=\frac{1}{\pi} \ln \frac{z-\Delta}{z+\Delta} .
$$

Note that $\operatorname{Im} h_{\Delta}(z) \approx 1$ if $|z|<\Delta$ and $\operatorname{Re} z \approx 0$. This means that compositions with $h_{\Delta}$ can be used to construct Herglotz functions that are suitable to bound regions with low values of $|z|$.

The admittance $Y=1 / Z$ and its associated Herglotz function $h_{\mathrm{Y}}(k)=\mathrm{i} Y(k) \eta_{0}$ are small for high-impedance surfaces. The composition $h_{\Delta}\left(h_{\mathrm{Y}}(k)\right)$ has the asymptotic expansion

$$
h_{\Delta}\left(h_{\mathrm{Y}}(k)\right) \sim \frac{2}{\pi} \mu_{\mathrm{s}} \Delta k d \quad \text { as } k \stackrel{\leftrightarrow}{\rightarrow} 0 .
$$

The identity (1) gives the $p=1$ sum rule

$$
\int_{0}^{\infty} \frac{\operatorname{Im} h_{\Delta}\left(h_{\mathrm{Y}}(k)\right)}{k^{2}} \mathrm{~d} k=\mu_{\mathrm{s}} d \Delta .
$$

As an example, consider the reflection in free space determined at the distance $d$ from a ground plane, i.e., $\mu_{\mathrm{s}}=1$ in the equations above. The admittance is infinite for $d / \lambda=$ $k d /(2 \pi)=n / 2$ and vanishes for $d / \lambda=1 / 4+n / 2$, where $n=0,1,2, \ldots$, see Fig. 4 . The structure is lossless so the admittance is purely imaginary where it is defined. This means that $\operatorname{Im}\left\{h_{\mathrm{Y}}(k)\right\}=0$ except at the singular points. The composition $h_{\Delta}\left(h_{\mathrm{Y}}(k)\right)$ is determined for $\Delta=1$, and it is noted that $h_{\Delta}\left(h_{\mathrm{Y}}(k)\right)=1$ for wavenumbers such that $\left|h_{\mathrm{Y}}(k)\right|<\Delta=1$ or equivalently $Y(k)<\eta_{0}$.

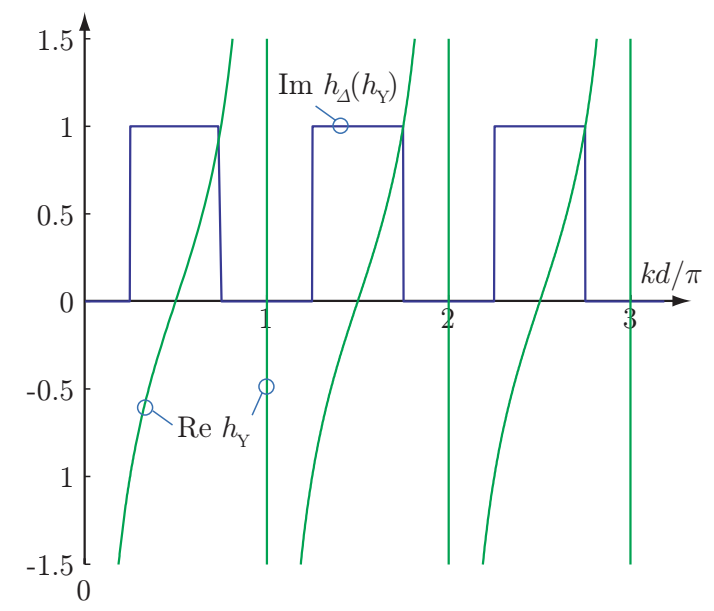

Fig. 4. Admittance function $h_{\mathrm{Y}}(k)$ and composition $h_{\Delta}\left(h_{\mathrm{Y}}(k)\right)$ with $\Delta=$ 1 the reflection of ground plane at distance $d$.

\section{Constitutive Relations}

Causality and passivity are also common assumptions in the constitutive relations used in electromagnetic theory. Consider for simplicity isotropic models for the (relative) permittivity, $\epsilon_{\mathrm{r}}(\omega)$, where $\omega$ denotes the angular frequency. Define the 
Herglotz function $h_{\epsilon}(k)=\omega \epsilon(\omega) / \omega_{0}$. It has the asymptotic expansions

$$
h_{\epsilon}(\omega) \sim\left\{\begin{array}{l}
\epsilon_{\mathrm{s}} \omega / \omega_{0}, \quad \text { as } \omega \hat{\longrightarrow} 0 \\
\epsilon_{\infty} \omega / \omega_{0}, \quad \text { as } \omega \hat{\rightarrow} \infty,
\end{array}\right.
$$

where $\epsilon_{\mathrm{S}}$ is the static (relative) permittivity, $\epsilon_{\infty}$ is the optical response, and $\omega_{0}>0$ a frequency parameter. Note that the low-frequency expansion changes to $\mathrm{i} \sigma / \omega_{0}$ in the case with a static conductivity. The identity (1) gives a $p=1$ sum rule for $\epsilon$ that is identical to the sum rule derived from the KramersKronig relations [18].

To construct an identity that is suitable for $\epsilon$-near zero materials, it is observed that this case is similar to the highimpedance surface considered in Sec. IV-C, i.e., it is desired to find constraints on low amplitudes of a Herglotz functions. The composition $h_{\Delta}\left(h_{\epsilon}(\omega)\right)$ has the asymptotic expansions [8]

$$
h_{\Delta}\left(h_{\epsilon}(\omega)\right) \sim\left\{\begin{array}{l}
\mathrm{i}, \quad \text { as } \omega \hat{\rightarrow} 0 \\
\frac{-2 \omega_{0} \Delta}{\omega \pi \epsilon_{\infty}}, \quad \text { as } \omega \hat{\longrightarrow} \infty,
\end{array}\right.
$$

and the $p=0$ identity (1) gives

$$
\begin{aligned}
& \int_{0}^{\infty} \operatorname{Im} h_{\Delta}\left(h_{\epsilon}(\omega)\right) \mathrm{d} \omega \\
& \quad=\int_{0}^{\infty} \frac{1}{\pi} \arg \left(\frac{\omega \epsilon(\omega)-\omega_{0} \Delta}{\omega \epsilon(\omega)+\omega_{0} \Delta}\right) \mathrm{d} \omega=\frac{\omega_{0} \Delta}{\epsilon_{\infty}} .
\end{aligned}
$$

This identity is transformed into a bound on $\epsilon$ where it is observed that the bound in lossy media is similar to the bound in [19] for $B \ll 1$ with $\epsilon_{\infty}=1$, see [8] for details.

As an example, consider the Drude model

$$
\epsilon(\omega)=1+\frac{1}{-\mathrm{i} \omega(0.01-\mathrm{i} \omega)},
$$

where $\omega$ is a dimensionless frequency variable, see Fig. 5. The permittivity is near zero for $\omega \approx 1=\omega_{0}$. The sum rule (23) is evaluated with $\Delta=0.5$. The integrand in (23) is depicted in Fig. $5 b$, where it is observed that it has most of its area in the region around $\omega \approx 1$, i.e., in the region where $|\epsilon(\omega)| \leq \Delta$. The amplitudes $\left|h_{\epsilon}(\omega)\right|$ and $|\epsilon(\omega)|$ are also included in the figure.

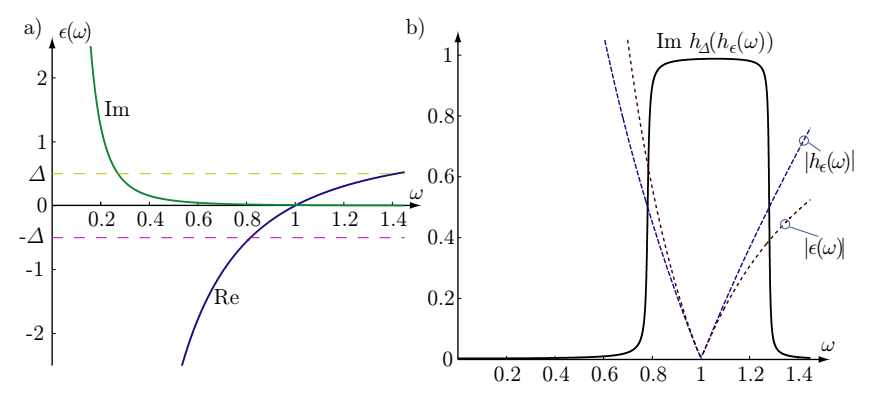

Fig. 5. Illustrations of the Drude model (24) with the sum rule (23). a) the permittivity $\epsilon(\omega)$. b) the integrand in the sum rule (23), $\operatorname{Im} h_{\Delta 1}=\operatorname{Im} h_{\Delta}\left(h_{1}\right)$, the function $\left|h_{\epsilon}\right|$, and the difference $|\epsilon|$ with $\Delta=0.5$.

\section{CONCLUSIONS}

It is shown that several sum rules in electromagnetic theory can be derived in a unified way using integral identities for Herglotz functions. The approach is illustrated with sum rules for the extinction cross section, antenna impedance and admittance, reflection and transmission coefficients, and dispersion of metamaterials.

\section{ACKNOWLEDGMENTS}

The support of the Swedish research council is gratefully acknowledged.

\section{REFERENCES}

[1] H. M. Nussenzveig, Causality and dispersion relations. London: Academic Press, 1972

[2] A. Bernland, M. Gustafsson, and A. Luger, "Sum rules and constraints on passive systems," 2010, to be submitted.

[3] A. H. Zemanian, Distribution theory and transform analysis: an introduction to generalized functions, with applications. New York: Dover Publications, 1987.

[4] K. N. Rozanov, "Ultimate thickness to bandwidth ratio of radar absorbers," IEEE Trans. Antennas Propagat., vol. 48, no. 8, pp. 12301234, Aug. 2000.

[5] C. Sohl, M. Gustafsson, and G. Kristensson, "Physical limitations on broadband scattering by heterogeneous obstacles," J. Phys. A: Math. Theor., vol. 40, pp. $11165-11182,2007$.

[6] C. R. Brewitt-Taylor, "Limitation on the bandwidth of artificial perfect magnetic conductor surfaces," Microwaves, Antennas \& Propagation, IET, vol. 1, no. 1, pp. 255-260, 2007.

[7] M. Gustafsson, C. Sohl, C. Larsson, and D. Sjöberg, "Physical bounds on the all-spectrum transmission through periodic arrays," $E P L$ Europhysics Letters, vol. 87, no. 3, p. 34002 (6pp), 2009. [Online]. Available: http://stacks.iop.org/0295-5075/87/34002

[8] M. Gustafsson and D. Sjöberg, "Sum rules and physical bounds on passive metamaterials," Lund University, Department of Electrical and Information Technology, P.O. Box 118, S-22100 Lund, Sweden, Tech. Rep. LUTEDX/(TEAT-7186)/1-19/(2010), 2010, http://www.eit.lth.se.

[9] M. Gustafsson, C. Sohl, and G. Kristensson, "Physical limitations on antennas of arbitrary shape," Proc. R. Soc. A, vol. 463, pp. 2589-2607, 2007.

[10] — , "Illustrations of new physical bounds on linearly polarized antennas," IEEE Trans. Antennas Propagat., vol. 57, no. 5, pp. 1319-1327, May 2009.

[11] M. Gustafsson, "Sum rule for the transmission cross section of apertures in thin opaque screens," Opt. Lett., vol. 34, no. 13, pp. 2003-2005, 2009.

[12] R. M. Fano, "Theoretical limitations on the broadband matching of arbitrary impedances," Journal of the Franklin Institute, vol. 249, no. 1,2 , pp. $57-83$ and $139-154,1950$.

[13] C. Sohl, M. Gustafsson, and G. Kristensson, "The integrated extinction for broadband scattering of acoustic waves," J. Acoust. Soc. Am., vol. 122, no. 6, pp. 3206-3210, 2007.

[14] E. M. Purcell, "On the absorption and emission of light by interstellar grains," J. Astrophys., vol. 158, pp. 433-440, 1969.

[15] C. Sohl, C. Larsson, M. Gustafsson, and G. Kristensson, "A scattering and absorption identity for metamaterials: experimental results and comparison with theory," J. Appl. Phys., vol. 103, no. 5, p. 054906, 2008.

[16] B. Ung and Y. Sheng, "Interference of surface waves in a metallic nanoslit," Optics Express, vol. 15, no. 3, pp. 1182-1190, 2007.

[17] D. Sjöberg, "Low frequency scattering by passive periodic structures for oblique incidence: low pass case," J. Phys. A: Math. Theor., 2009, accepted for publication.

[18] L. D. Landau, E. M. Lifshitz, and L. P. Pitaevskiı̌, Electrodynamics of Continuous Media, 2nd ed. Oxford: Pergamon, 1984.

[19] Ø. Lind-Johansen, K. Seip, and J. Skaar, "The perfect lens on a finite bandwidth," J. Math. Phys., vol. 50, p. 012908, 2009. 